\title{
Crystal structure and characterization of pyrroloquinoline quinone disodium trihydrate
}

\author{
Kazuto Ikemoto ${ }^{*}$, Hitoshi Sakamoto and Masahiko Nakano
}

\begin{abstract}
Background: Pyrroloquinoline quinone (PQQ), a tricarboxylic acid, has attracted attention as a growth factor, and its application to supplements and cosmetics is underway. The product used for these purposes is a water-soluble salt of PQQ disodium. Although in the past, $\mathrm{PQQ}$ disodiumpentahydrates with a high water concentration were used, currently, low hydration crystals of PQQ disodiumpentahydrates are preferred.

Results: We prepared a crystal of PQQ disodium trihydrate in a solution of ethanol and water, studied its structure, and analyzed its properties. In the prepared crystal, the sodium atom interacted with the oxygen atom of two carboxylic acids as well as two quinones of the PQQ disodium trihydrate. In addition, the hydration water of the prepared crystal was less than that of the conventional PQQ disodium crystal. From the results of this study, it was found that the color and the near-infrared (NIR) spectrum of the prepared crystal changed depending on the water content in the dried samples.

Conclusions: The water content in the dried samples was restored to that in the trihydrate crystal by placing the samples in a humid environment. In addition, the results of X-ray diffraction (XRD) and X-ray diffraction-differential calorimetry (XRD-DSC) analyses show that the phase of the trihydrate crystal changed when the crystallization water was eliminated. The dried crystal has two crystalline forms that are restored to the original trihydrate crystals in 20\% relative humidity (RH). This crystalline (PQQ disodium trihydrate) is stable under normal environment.
\end{abstract}

\section{Introduction}

Pyrroloquinoline quinone (PQQ; 4,5-dihydro-4,5-dioxo1 H-pyrrolo[2,3-f]quinoline- 2,7,9-tricarboxylic acid) (Figure 1a) is a water-soluble quinone that was first identified as a non-covalently prosthetic group in some bacterial glucose- or alcohol dehydrogenases [1,2]. It is interesting to note that trace amounts of PQQ have been found not only in microorganisms but also in humans and in the organs and tissues of rats, with the highest amount being found in human milk [3,4]. In addition, trace amounts of PQQ have also been found in daily foods and beverages [5,6]. In recent years, PQQ has been receiving considerable attention owing to its several interesting physiological functions [7,8]. PQQ stimulates mitochondrial biogenesis through cAMP response element-binding protein phosphorylation and an increase in PGC- $1 \alpha$ expression $[9,10]$. In addition, PQQ promotes the regeneration of peripheral and central nerves. From

\footnotetext{
* Correspondence: kazuto-ikemoto@mgc.co.jp

Niigata Research Laboratory, Mitsubishi Gas Chemical Company, Inc, 182 Tayuuhama Shinwari, Kita-ku, Niigata, Japan
}

the results of the in vitro experiment, it was found that PQQ enhances the nerve growth factor (NGF)-a neurotrophic factor responsible for the maintenance and development of peripheral nerves [11]. Further, the regeneration of a transected sciatic nerve in an in vivo rat model was demonstrated [12]. A recent study suggests that PQQ protects against secondary damage by attenuating the inducible nitric oxide synthase (iNOS) expression following a primary physiological injury to the spinal cord [13].

Previous studies showed that PQQ and reduced PQQ are antioxidative in nature $[14,15]$. Their antioxidative function was observed in culture cells and rats [16,17]. PQQ disodium of an ionic material is often used as a water-soluble salt because the tricarboxylic acid type has low solubility. In many cases, organic molecules exist in a crystalline state in several different forms such as polymorphs or hydrates. The crystalline form of an organic molecule is important from the viewpoint of its application in food and pharmaceutical products. However, the polymorphs of PQQ disodium crystalline have not been studied. Thus far, the crystal structures of an acetone adduct [2], a metal complex [18],
() Chemistry Central

(C) 2012 Ikemoto et al.; licensee Chemistry Central Ltd. This is an Open Access article distributed under the terms of the Creative Commons Attribution License (http://creativecommons.org/licenses/by/2.0), which permits unrestricted use, distribution, and reproduction in any medium, provided the original work is properly cited. 

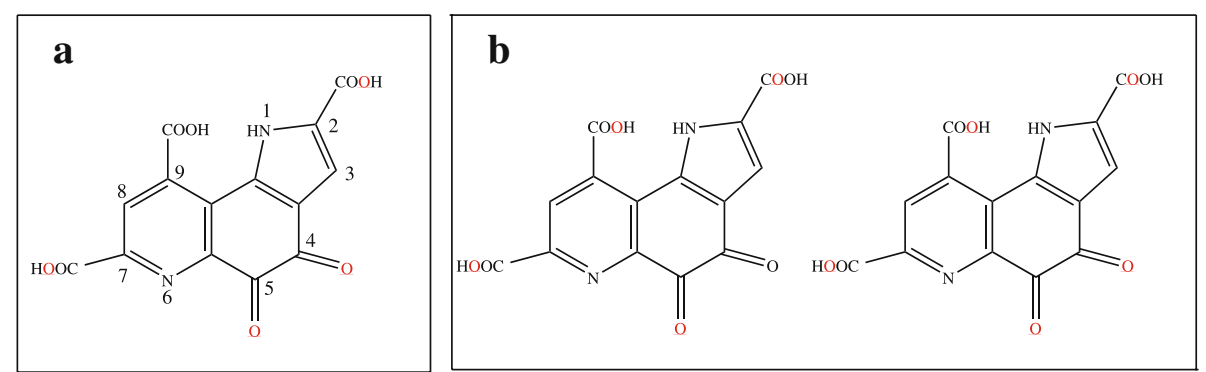

Figure 1 Chemical structure of PQQ. Sodium interacts with the oxygen present at the underlined position. (a) PQQ disodium trihydrate 2- and 7-COOH groups take anion forms (2- and 7-COO-). (b) PQQ disodium pentahydrate. Two crystallographically independent conformation PQQ.

an ester [19], and PQQ disodium pentahydrate [20] have been reported.

A single crystal of PQQ disodium pentahydrate was obtained by the slow evaporation of phosphate buffer at $20^{\circ}$ C. However, the crystal of PQQ disodium pentahydrate obtained by this method is unstable because it contains a large amount of water. Therefore, owing to this drawback, the abovementioned method is not suitable for the industrial manufacture of crystals of PQQ disodium pentahydrate. On the other hand, a powder of PQQ disodium was industrially manufactured by adding ethanol (poor solvent) to a water solution of PQQ disodium [21]. However, this product, which included ethanol, had low crystallinity.

We successfully prepared PQQ disodium trihydrate with low water content. The structure of the prepared crystal was analyzed by single crystal X-ray diffraction.

Further, we studied the effect of the water content on the crystalline form, color, and near-infrared (NIR) spectrum of the prepared crystal.

\section{Results and discussion}

Single crystal analysis

A molecule of PQQ was separated from the culture of a microbe, and PQQ trisodium was salted out at neutral
$\mathrm{pH}$. The $\mathrm{pH}$ of the suspension of PQQ trisodium in ethanol/water was reduced, and PQQ disodium was crystallized. The board-shaped crystal of PQQ disodium (Figure 2) exhibits high crystallinity; the result of single crystal X-ray diffraction analysis is shown in Figure 2, and Table 1 summarizes the supporting information. The obtained crystal is PQQ disodium trihydrate (type I), and it does not include ethanol. With regard to the crystals prepared by the conventional ethanol precipitation method, it is found that ethanol is present in the sediment and that the crystals exhibit poor crystallinity. As shown in Figure 2, $\mathrm{Na}^{+}-1$ forms ionic bonds with the oxygen atoms of the 7-COO- and 5-CO-groups, and $\mathrm{Na}$ ${ }^{+}-2$ forms an ionic bond with an oxygen atom of the 2-COO- group in PQQ. In addition, $\mathrm{Na}^{+}-2$ also forms a bond with the oxygen atom of 4-CO of the neighboring molecule. In PQQ disodium trihydrate, the 2- and 7-COOH groups take anion forms (2- and 7-COO-). Moreover, such bonding is not observed in 9-carboxylic acid (Figure 1a). A crystal of PQQ disodium pentahydrate comprises two types of molecules of PQQ (Figure $1 \mathrm{~b}$ ); and thus far, it has been observed that only one of those quinones did not form sodium and an ionic bond. The difference in the type of molecules leads to a
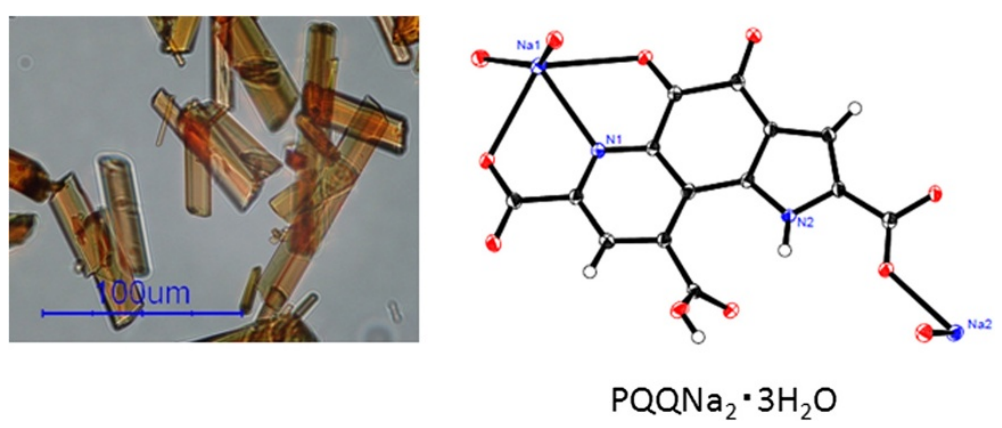

Figure 2 Left: PQQ disodium trihydrate crystal observed under a microscope. Right: Molecular structure of PQQ disodium trihydrate (ORTEP). 
Table 1 Crystal data for PQQdisodium trihydrate

\begin{tabular}{ll}
\hline Empirical Formula & $\mathrm{C}_{14} \mathrm{H}_{10} \mathrm{~N}_{2} \mathrm{Na}_{2} \mathrm{O}_{11}$ \\
\hline Formula Weight & 428.22 \\
\hline Crystal Color & Habit red, block \\
\hline Crystal Dimensions & $0.100 \times 0.100 \times 0.100 \mathrm{~mm}$ \\
\hline Crystal System & triclinic \\
\hline Lattice Parameters & Primitive, a $=7.6087(2) \AA, \mathrm{b}=10.0963(2) \AA$, \\
& $\mathrm{C}=11.4337(2) \AA, \mathrm{a}=72.858(5)^{\circ}, \beta=88.015(7)^{\circ}{ }^{\circ}$, \\
\hline Space Group & $\mathrm{Y}=82.627(6)^{\circ}, \mathrm{V}=832.37(4) \AA 3^{\circ}$ \\
\hline Z value & 2 \\
\hline Dcalc. & $1.708 \mathrm{~g} / \mathrm{cm}^{3}, \mathrm{~F} 000436.00$ \\
\hline
\end{tabular}

difference in the lattice multiplier. Furthermore, the difference resulted in the production of our crystal at $3.195 \AA$ with $3.607 \AA$, as well as resulted in the formation of pentahydrates in the interval between the shortest $\pi$ planes.

The crystal obtained was nothing but the PQQ disodium crystal, but with a low amount of water. This low hydration is effective in suppressing the growth of microbe and increasing the stability of the material when it is used in food and drugs.

\section{Drying and moisture absorption}

The PQQ disodium trihydrate crystal contains 12.7\% water. The water included in the crystal can be eliminated by vacuum or heat drying. The crystal prepared in this study had a water content of $26.5 \%$, and water was even found on the surface of the crystal. However, after this crystal was subjected to vacuum drying, its water content decreased drastically to only $0.7 \%$. It was found that the color of the crystal changed considerably from red to dark brown with a decrease in the water content (Figure 3). The obtained crystal was subjected to colorimetric analysis; this analysis did not have an effect on the color of the crystal and it reduced the chroma. The results of this analysis showed that the change in the color of the crystal was linear.

Recently, near-infrared (NIR) analysis has been used for the quality control of food and drugs. An important point to note here is that the results of this method of analysis are also strongly influenced by the water content in a crystal. From the NIR spectrum of a crystal, it was found that a broad peak became sharp with a decrease in the water content (see Figure 3). The water content of the samples converged to approximately $12 \%$ when the samples were subjected to a humidity of $75 \% \mathrm{RH}$ at $40^{\circ} \mathrm{C}$ for 1 day. After moisture absorption, the color and the NIR spectra of all the samples were identical to those of the original trihydrate crystal. It is interesting to note that a sample whose water content was $26.5 \%$ higher than that of the trihydrate crystal showed a water content of only $12 \%$. This result indicated that the water outside the crystal evaporated and that the crystalline water remained. It is easy to change the weight of a very dry sample in a normal environment because the dried sample now becomes hygroscopic in nature.

\section{Water content and powder $\mathrm{X}$-rays analysis}

Techniques for carrying out structure determination directly from powder X-ray diffraction (XRD) data clearly provide the structural properties of a polycrystalline product in solid-state transformation. We studied the structure of the crystal of PQQ disodium trihydrate
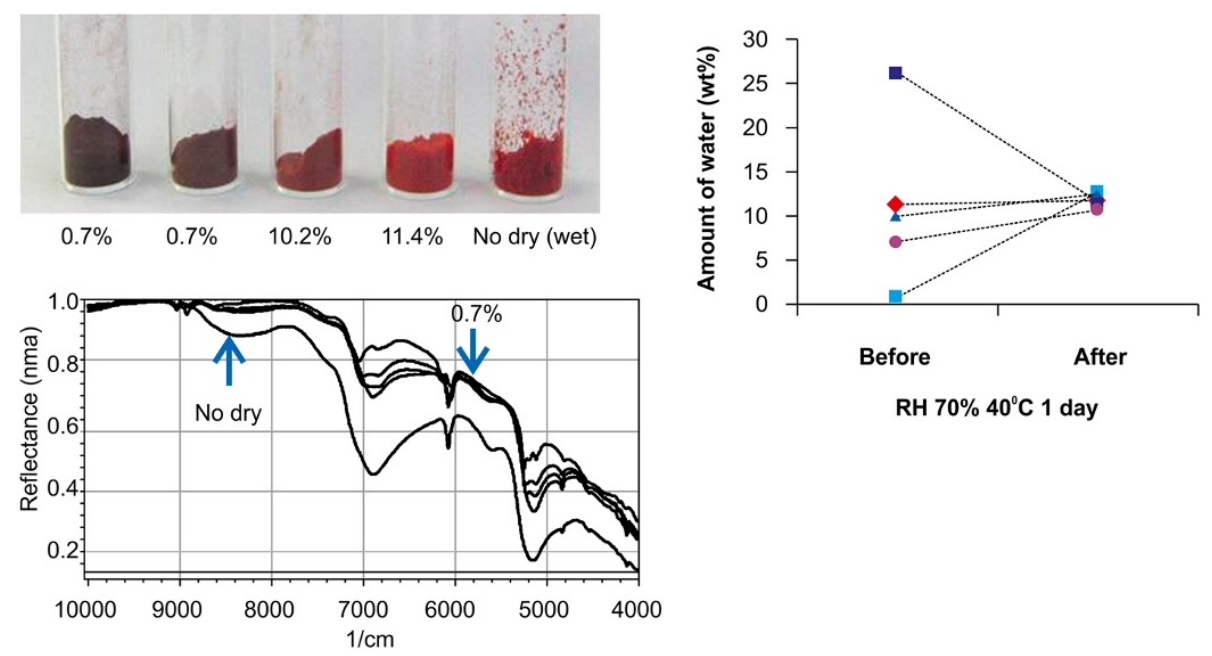

Figure 3 Samples with different water content prepared by drying PQQ disodium trihydrate. Images of the samples with different water content. Near-infrared (NIR) spectra of dried samples. All the samples with different water content show a water content of around $12 \%$ after being subjected to a relative humidity $(\mathrm{RH})$ of $75 \%$ at $40^{\circ} \mathrm{C}$ for 1 day. 


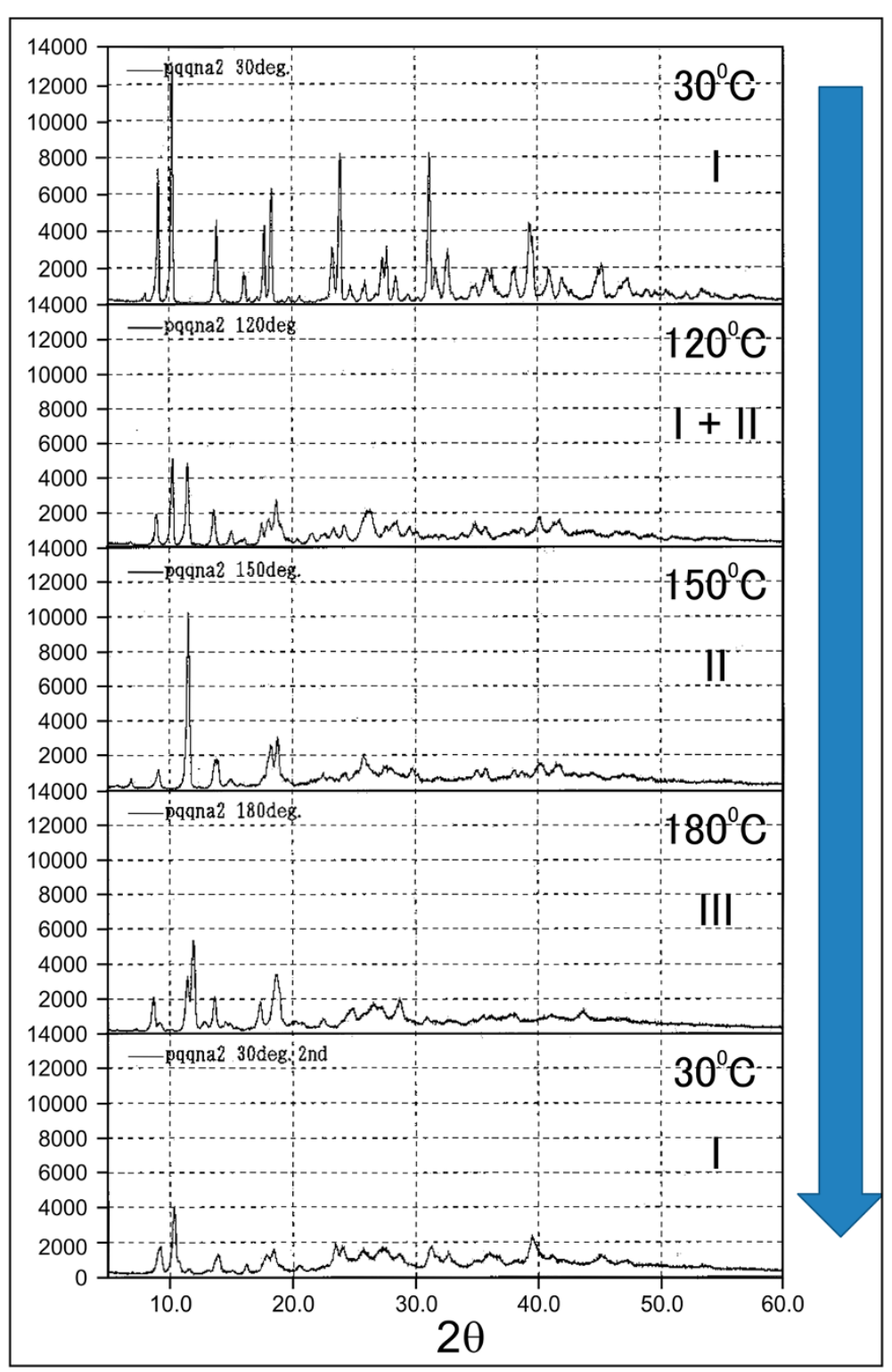

Figure 4 Powder X-ray diffractometry (XRD) of PQQ disodium trihydrate in air. The type I trihydrate crystal changed to the type II trihydrate crystal at $150^{\circ} \mathrm{C}$, which in turn changed to the type III crystal at $180^{\circ} \mathrm{C}$. The crystal formed at $120^{\circ} \mathrm{C}$ is a mixture of type I and type II crystals. $2 \theta$ =type I: $9.1,10.3,13.8,17.7,18.3,24.0,27.4$, type II: 11.4, 13.5, 18.0, 18.7, 26.0, 28.5, type III: 7.0, 11.5, 12.0, 17.4, 18.7.

(type I) by XRD when it was subjected to the drying process. The temperature was continuously changed to examine a decrease in the water content and a change in the crystal structure.

The result of the effect of the variation in temperature on the water content is shown in Figure 4 . The water included in the crystal can be eliminated by a general drying method. The crystal was subjected to crystallographic analysis during which the water content in the crystal changed with drying. In addition, the temperature was changed in the air to examine a decrease in the water content and a change in the crystal structure. In the XRD spectrum of the trihydrate crystal (type I), new peaks were observed at $120^{\circ} \mathrm{C}$ and the crystal also exhibited a new crystalline form. The type I crystal disappeared at $150^{\circ} \mathrm{C}$, and a new crystal (type II) was formed. The type II crystal is capable of exhibiting higher symmetry than the type I crystal because its XRD spectrum shows few peaks. Furthermore, at temperatures higher than $180^{\circ} \mathrm{C}$, a type III crystal was formed. It was observed that a peak of the type I crystal appeared when the temperature decreased to $30^{\circ} \mathrm{C}$ for $1 \mathrm{~h}$ under atmospheric conditions. 


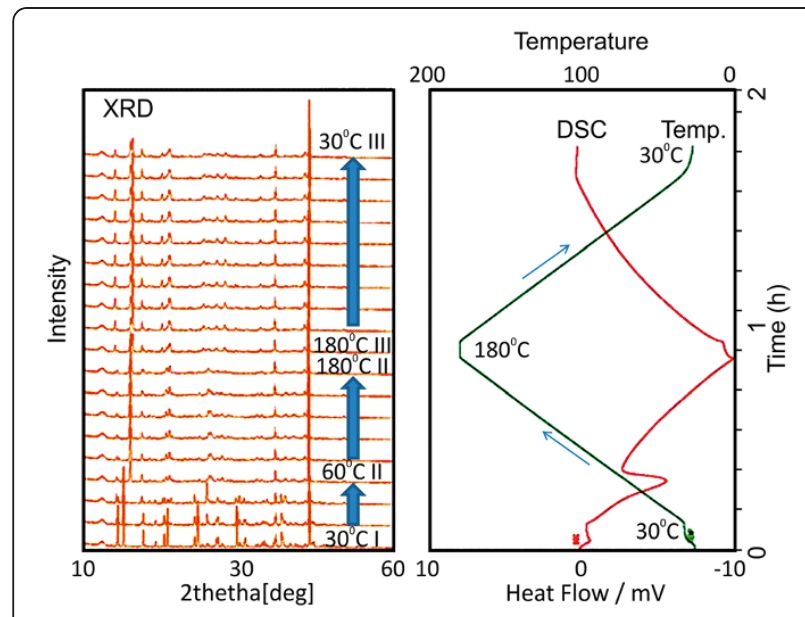

Figure 5 XRD-DSC (left, XRD; right, DSC, temperature).

Temperature was changed from $30^{\circ} \mathrm{C}$ to $180^{\circ} \mathrm{C}$ and back to $30^{\circ} \mathrm{C}$ $\left(3^{\circ} \mathrm{C} / \mathrm{min}\right)$, and the second phase conversion occurred at $180^{\circ} \mathrm{C}$.

In this study, the samples with different water content were measured by XRD. The samples with a water content of $10.2-26.5 \%$ were type I crystals. The samples with a water content of $7.0 \%$ were a mixture of type I and type II crystals, such as the sample obtained at $120^{\circ}$ C. The samples with a water content of $0.7 \%$ were only type II crystals. After treatment in a humid environment, all the samples became type I crystals and contained around $12 \%$ water. As in the case of the color of the crystal, its structure was restored by the water content. The phase of the crystal undergoes conversion. Furthermore, simultaneous X-ray diffraction-differential scanning calorimetry (XRD-DSC) was performed for detailed analysis of the crystal phase. The temperaturedependent measurement of PQQ disodium trihydrate in nitrogen (no humidity) is shown in Figure 5. The graph shown on the right depicts the DSC result of a measurement (temperature, DSC curve), and the graph shown on the left depicts the occasional XRD profile. Temperature was changed from $30^{\circ} \mathrm{C}$ to $180^{\circ} \mathrm{C}$ and back to $30^{\circ} \mathrm{C}\left(3^{\circ} \mathrm{C} / \mathrm{min}\right)$ in nitrogen. The XRD spectrum with heat absorption on the DSC curve at $40-85^{\circ} \mathrm{C}$ in the temperature-increasing process was changed, and the crystalline water was eliminated.

Amount of heat can be calculated from DSC curve. The analysis is shown below.

In heat absorption, $187 \mathrm{~J} / \mathrm{g}$, the peak top temperature was $73^{\circ} \mathrm{C}$, and crystal water was eliminated. A slight change of DSC was measured at $180^{\circ} \mathrm{C}$, and the conversion in the phase occurred at $180^{\circ} \mathrm{C}$. In the subsequent low-temperature process, the absorption of the heat and a change in the XRD profile were not observed. The peak obtained after the DSC of the dehydration of the crystalline water was in good agreement with that of the type II crystal obtained from the type I crystal of trihydrate. Furthermore, the type II crystal was changed to type III crystal at $180^{\circ} \mathrm{C}$. The mixture of type I and type II crystals could be observed in air, but not in nitrogen. The crystal structure did not change under dry condition. When the temperature of the type III crystal was lowered to room temperature under nitrogen atmosphere, the structure was maintained. However, the type III crystal structure changed to type I in the air.

The type II crystal is an anhydride; the experiment performed in this study shows that the phase of the type II crystal changed at $180^{\circ} \mathrm{C}$, at which point a new type III crystal was formed, with a structure that differed from that of the type II crystal. The hydration of the type III crystal at $35^{\circ} \mathrm{C}$ was measured by XRD-DSC in a moisture absorption process.

The humidity was increased from 0 to $90 \% \mathrm{RH}$, and the sample was measured. The result of this measurement is shown in Figure 6. A peak in the DSC curve of water absorption was observed when the humidity was increased to $20 \% \mathrm{RH}$. This peak lasted for approximately $130 \mathrm{~min}$, and the crystallization was observed when the XRD profile gradually changed. When the humidity was increased to $30 \% \mathrm{RH}$, change in DSC and in the XRD profile were not observed. It was inferred that the type III crystal transformed to the type I trihydrate crystal. The calorific value of water absorption was $177 \mathrm{~J} / \mathrm{g}$, which shows the type I of trihydrate crystal is $75.8 \mathrm{~kJ} / \mathrm{M}$ more stable than type III in normal air.

First, we thought that the change in the crystalline form advanced from trihydrates to mono- or di-hydrates in the type II crystal. It was thought that due to high absorbency, type III crystal was not observed in normal dry air. However, phase transition occurs in the type II crystal after a dehydration process on XRD-DSC in nitrogen. Moreover, the type II crystal was transformed to

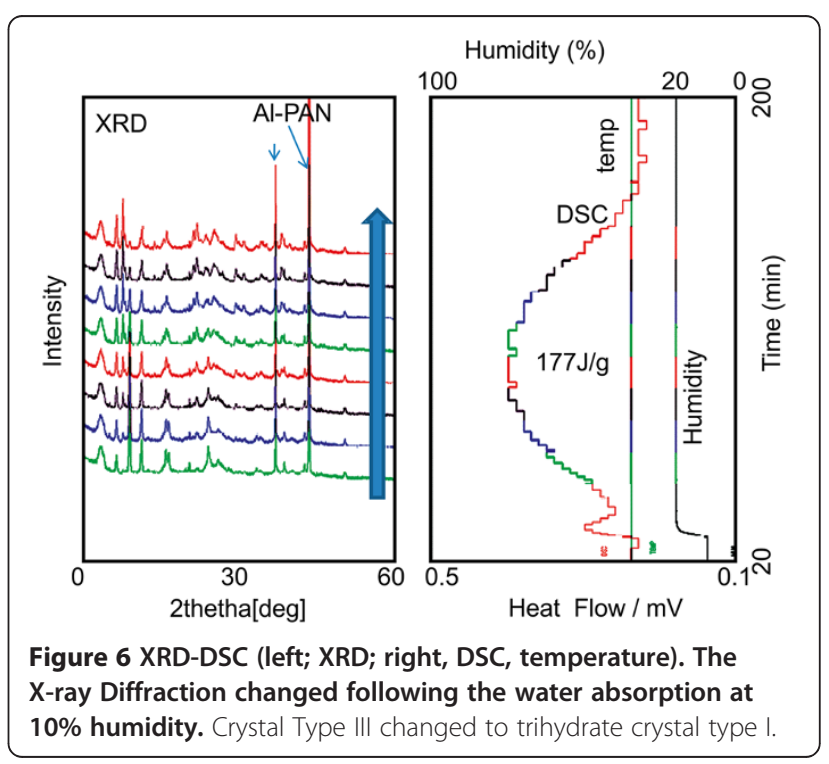




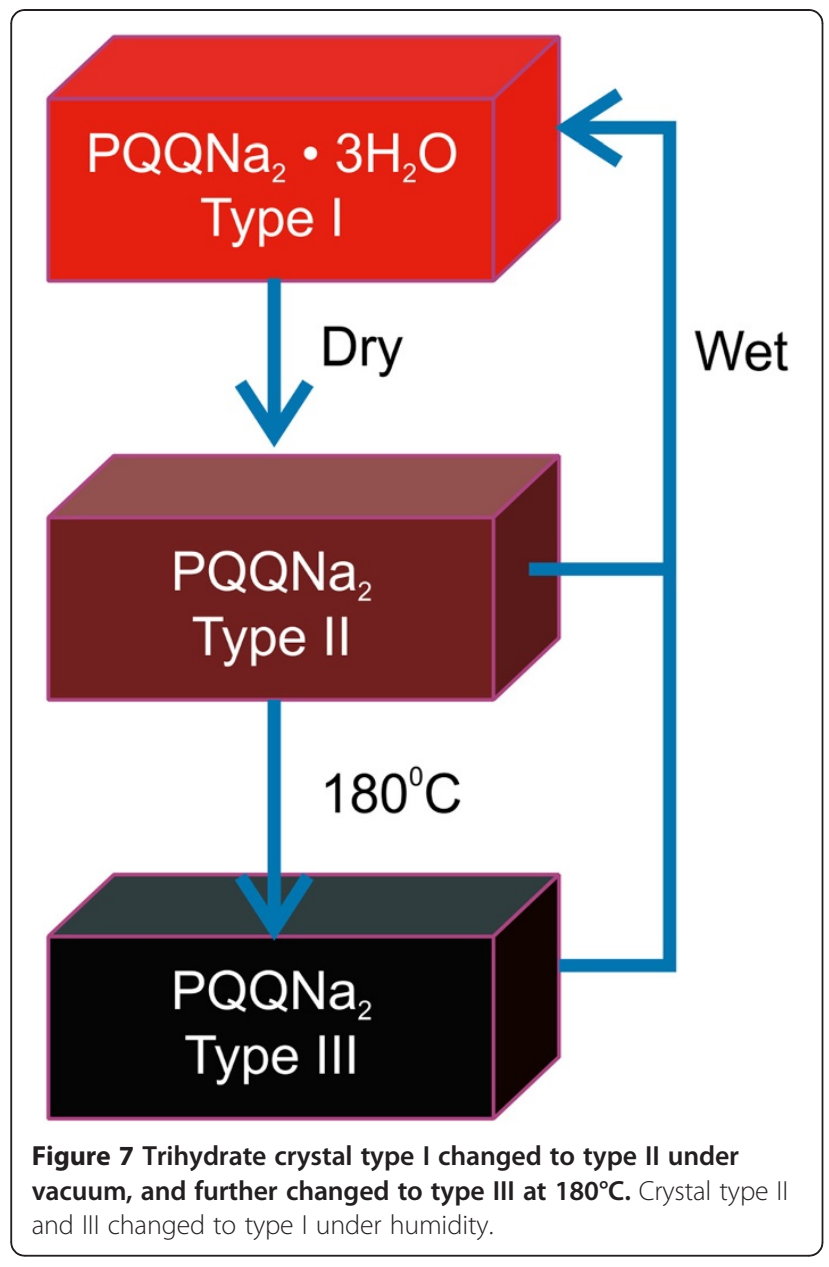

a type III crystal at a high temperature of $180^{\circ} \mathrm{C}$. In other words, the type II crystal is an anhydride crystal, and the type III crystal is an anhydrous crystal. A mixture of a type I and type II crystal can be obtained in air with humidity. Details on non-aqueous crystal structures are not yet understood.

We expected that on elimination of the water, the crystals would become either amorphous or porous. However, contrary to our expectation, the crystals were not amorphous. The surface area of these crystals was measured by nitrogen absorption. From the results of this measurement, it was found that the surface area of all the crystals was less than the BET surface area of $2 \mathrm{~m}^{2} / \mathrm{g}$. This result shows that the crystal structures are not porous and that they change considerably with the removal of water.

We also found that the water intercalated in to a crystal to result in the formation of trihydrate. The following conclusions can be drawn from the abovementioned results (Figure 7). The color, the NIR spectrum attributed to drying, and the structure of the type II trihydrate crystals change.
Furthermore, phase transition of the type II crystal occurs at $180^{\circ} \mathrm{C}-\mathrm{a}$ temperature at which the type III crystal is formed. The crystal can either be of type I, a mixture of type I and type II, and type II, depending on the humidity in air. The type III crystal can also be obtained at room temperature by reducing the phase transition temperature of the type II crystal in the dry state. The trihydrates were found to be stable in a humid environment, whereas the type II and type III crystals were unstable in this environment in that they both returned to their original phase. Trihydrate crystals can easily absorb as much as $20 \%$ water from a humid environment, contributing to their stability under humidity. Therefore, a trihydrate crystal exhibits suitable properties such that it can be used as a commercial product.

\section{Summary}

A single crystal of PQQ disodium trihydrate was prepared. Two carboxylic acid and two quinone form ionic bonds with oxygen and sodium in this crystal. The water content of this crystal had a significant effect on the color and the change in the NIR absorption spectrum; the prepared crystal was found to undergo phase transition at different temperatures. This crystal exhibits a crystalline form that $180^{\circ}$ or more different from that of an anhydride. The dried sample becomes a trihydrate crystal at $20 \%$ humidity, with the color and NIR spectrum same as trihydrate crystals. In case of this crystal, trihydrates are stable and their absorbency is low. For their analysis and stability of their color, extensive drying should be avoided. This crystalline (PQQ disodium trihydrate) is stable under normal environment.

\section{Experimental methods}

\section{Preparation of PQQ disodium crystals}

The PQQ was prepared by fermentation and was purified by column chromatography. The PQQ trisodium solid was precipitated by adding $\mathrm{NaCl}$ at $\mathrm{pH}$ 7.5. A suspension of this solid was obtained with the addition of a $50 \%$ by volume ethanol-water solution. Then, hydrochloric acid was slowly added to this suspension, following which the suspension was crystallized at $\mathrm{pH} 3.5$, and a PQQ disodium crystal was obtained. The samples with different content of crystallization water were prepared under different vacuum conditions.

\section{Water content analysis}

The sample was heated to $180^{\circ} \mathrm{C}$, and the water that was eliminated from the crystal was analyzed by the Karl Fischer method using a Metrohm 831 KF coulometer. 


\section{Single crystal X-ray structure analysis}

Rigaku R-AXIS RAPID, radiation $\mathrm{Cu}-\mathrm{K} \alpha$ voltage/current: $40 \mathrm{kV} / 30 \mathrm{~mA}, 23.0^{\circ} \mathrm{C}$. Crystallography data have been deposited additional files, Additional file 1: Check cif report, Additional file 2: Original X-ray analysis data by CIF format.

Powder X-ray diffractometry (XRD)

M18XCE, manufactured by MAC Science, Co. Ltd.

$\mathrm{Cu}-\mathrm{K} \alpha 40 \mathrm{kV} / 100 \mathrm{~mA}$, divergence slit: $1^{\circ}$, scattering slit: $1^{\circ}$, receiving slit: $0.3 \mathrm{~mm}$, scan speed: $4.000^{\circ} / \mathrm{min}$, sampling width: $0.02^{\circ}$.

XRD-DSC

Rigaku SmartLab (sample horizontal model multipurpose X-ray diffraction)

High-speed one-dimensional detector D/teX Ultra

$\mathrm{X}$-ray source $\mathrm{Cu}-\mathrm{K} \alpha(40 \mathrm{kV} / 50 \mathrm{~mA})$ with a scanning speed of $5^{\circ} / \mathrm{min}$

$0 \% \mathrm{RH}, 30-180^{\circ} \mathrm{C}\left(3^{\circ} \mathrm{C} / \mathrm{min}\right)$ Step $2: 35^{\circ} \mathrm{C}, 0-90 \% \mathrm{RH}$ atmosphere dry-wet $\mathrm{N}_{2}$.

\section{Additional files}

Additional file 1: Check CIF/PLATON report.

Additional file 2: Original X-ray analysis data.

\section{Competing interests}

The authors declare that they have no competing interests.

\section{Authors' contribution}

$\mathrm{KI}$ has coordinated the study, carried out the experimental work and drafted the manuscript. HS contributed the synthesis of powder of different water content. MN and KI discussed and analyzed the results. All authors have read and approved the final manuscript.

Received: 12 March 2012 Accepted: 6 June 2012

Published: 19 June 2012

\section{References}

1. Duine JA, Frank JJ, Jongejan JA: Glucose dehydrogenase from Acinetobacter calcoaceticus. A 'quinoprotein'. FEBS Lett 1979, 108:443-446.

2. Salisbury SA, Forrrest HS, Gruse WBT, Kennard O: A novel coenzyme from bacterial primary alcohol dehydrogenases. Nature 1979, 280:843-844.

3. Kumazawa T, Seno H, Urakami T, Matsumato T, Suzuki O: Trace levels of $\mathrm{PQQ}$ in human and rat samples detected by gas chromatography/mass spectroscopy. Biochim Biophys Acta 1992, 1156:62-66.

4. Mitcell $A E$, Johnes AD, Mercer RS, Rucker RB: Characterization of PQQ amino acid derivatives by electrospray ionization mass spectrometry and detection in human milk. Anal Biochem 1999, 269:317-325.

5. Kumazawa T, Sato K, Seno H, Ishii A, Suzuki O: Levels of PQQ in various foods. Biochem J 1995, 307:331-333.

6. Noji N, Nakamura T, Kitahata N, Taguchi K, Kudo T, Yoshida S, Tsujimoto M, Sugiyama T, Asami T: Simple and sensitive method for PQQ (PQQ) analysis in various foods using liquid chromatography/electrosprayionization tandem mass spectrometry. J Agric Food Chem 2007, $55: 7258-7263$

7. Stites TE, Mitchel AE, Rucker RB: Physiological importance of quinoenzymes and o-quinone family of cofactors. J Nutr 2000, 130:719-727.

8. Rucker R, Chowanadisai W, Nakano M: Potential physiological importance of PQQ. Altern. Med. Rev. 2009, 14:268-277.
9. Bauerly KA, Storms DH, Harris CB, Hajizadeh S, Sun MY, Cheung CP, Satre MA, Fascetti AJ, Tchaparian E, Rucker RB: PQQ nutritional status alters lysine metabolism and modulates mitochondrial DNA content in the mouse and rat. Biochim Biophys Acta 2006, 1760:1741-1748.

10. Chowanadisai W, Bauerly KA, Tchaparian E, Wong A, Cortopassi GA, Rucker RB: PQQ stimulates mitochondrial biogenesis through cAMP response element-binding protein phosphorylation and increased PGC-1alpha expression. J Biol Chem 2010, 285:142-152.

11. Yamaguchi K, Sasano A, Urakami T, Tsuji T, Kondo K: Stimulation of nerve growth factor production by $\mathrm{PQQ}$ and its derivatives in vitro and in vivo. Biosci. Biotech. Biochem. 1993, 57:1231-1233.

12. Liu S, Li H, Yang J, Peng H, Wu K, Liu Y, Yang J: Enhanced rat sciatic nerve regeneration through silicon tubes filled with PQQ. Microsurgery 2005, 25:329-337.

13. Hirakawa A, Shimizu K, Fukumitsu H, Furukawa S: Pyrroloquinoline quinone attenuates iNOS gene expression in the injured spinal cord. Biochem Biophys Res Commun 2009, 378:308-312.

14. He K, Nukada H, Urakami T, Murphy MP: Antioxidant and pro-oxidant properties of pyrroloquinoline quinone (PQQ); implications for its function in biological system. Biochem Pharmacol 2003, 65:67-74.

15. Ouch A, Nakano M, Nagaoka S, Mukai K: Kinetic of the antioxidant activity of pyrroloquinolinequinolinol $(\mathrm{PQQH} 2$, a reduced form of pyrroloquinoline quinone) in micellar solution. J Agric Food Chem 2009, 57:450-457.

16. Hara $H$, Hiramatsu $H$, Adachi T: Pyrroloquinoline quinone is a potent neuroprotective nutrient against 6-hydroxidopamine induced neurotoxicity. Neurochem Res 2007, 32:489-495.

17. Ohwada K, Takeda H, Yamazaki M, Isogaki H, Nakano M, Shimomura M, Fukui K, Urano S: Pyrroloquinoline quinone (PQQ) prevents cognitive deficit caused by oxidative stress in rats. J. Clin. Biochem. Nutr. 2008, 42:29-34.

18. Nakamura N, Kohzuma T, Kuma H, Suzuki S: Synthetic and structural studies on copper (II) complexes containing coenzyme PQQ and terpyridine. Inorg Chem 1994, 33:1594-1599.

19. Itoh S, Ogino M, Fukumi Y, Murao H, Komatsu M, Ohshiro Y, Inoue T, Kai Y, Kasai N: C-4 and C-5 adducts of cofactor PQQ (pyrroloquinolinequinone). Model studies direct toward the action of quinoprotein methanol dehydrogenase. J Am Chem Soc 1993, 115:9960-9967.

20. Ishida T, Doi M, Tomita K, Hayashi H, Inoue M, Urakami T: Molecular and crystal structure of PQQ (methoxatin), a novel coenzyme of quinoproteins: extensive stacking character and metal ion interaction. J Am Chem Soc 1989, 111:6822-6828.

21. Urakami M, Sugamura K, Yashima K: Method for recovering and purifying pyrroquinolinequinone. Japan: Industrial Property Libraly; 1988. http://www.ipdl.inpit.go.jp/homepg_e.ipdl.

doi:10.1186/1752-153X-6-57

Cite this article as: Ikemoto et al:: Crystal structure and characterization of pyrroloquinoline quinone disodium trihydrate. Chemistry Central Journal 2012 6:57.

\section{Publish with ChemistryCentral and every scientist can read your work free of charge \\ "Open access provides opportunities to our colleagues in other parts of the globe, by allowing anyone to view the content free of charge." W. Jeffery Hurst, The Hershey Company. \\ - available free of charge to the entire scientific community \\ - peer reviewed and published immediately upon acceptance \\ - cited in PubMed and archived on PubMed Central \\ - yours - you keep the copyright \\ Submit your manuscript here: \\ http://www.chemistrycentral.com/manuscript/ \\ ChemistryCentral}

